\title{
Interactive Design and Simulation Platform for Flight Vehicle Systems Development
}

\author{
Hugh H. T. Liu* \\ University of Toronto, Toronto, Ontario, M3H 5T6, Canada \\ Holger Berndt ${ }^{\dagger}$ \\ Universität Stuttgart, Stuttgart, Germany
}

\begin{abstract}
The need for integrated systems development arises from complexity of a system. This paper presents an interactive design and simulation platform for flight vehicle systems development. Its "connect-and-play" capability and adaptability enable "on-line" interaction between design and simulation during the integrated development. As a case study, the implementation of the proposed platform and an aircraft flight control system development example are demonstrated on an experimental test bed including a realtime systems simulator and a flight training device.
\end{abstract}

\section{Introduction}

The engineering systems development process typically has a waterfall view as if the different development stages were performed chronologically and independent to each other, when in reality they are extensively interrelated and interconnected. Moreover, a system often consists of multiple subsystems and components that are also interacting or even have conflicting characteristic features. Therefore, the entire development relies on iterative cycles between design modifications and integration and testing verifications, until the final design "converges". Unfortunately, this also makes the design process time-consuming and fragile: A slight change may require a completely new cycle of redesign. The need for an integrated development process arises from complexity, such as in many engineering intensive

\footnotetext{
*Associate Professor, Institute for Aerospace Studies (UTIAS), 4925 Dufferin Street, AIAA Member

${ }^{\dagger}$ Exchange Research Intern to the UTIAS, Institut für Flugmechanik und Flugregelung
} 
applications from automotive and aerospace industry. A research program entitled Software Enabled Control (SEC) is initiated to integrate multi-modal, coordinated operation of subsystems, and enable large-scale distribution of control. ${ }^{1}$ Under this program, an open control platform (OCP) is being developed to integrate control technologies and resources. ${ }^{2,3}$ The emerging field of multi-paradigm modeling addresses the directions of research in model abstraction, transformation, multi-formalism modeling, and meta-modeling. ${ }^{4}$

One of the key requirements for an integrated development process is the "plug-and-play" capability. It enables decoupled component (subsystem) design and integrated testing based on the same model. Therefore, design decisions are gradually finalized through iterations between component-level and system-level development. From complex control systems development point of view, ${ }^{5}$ advocate the computer automated multi-paradigm modeling. ${ }^{6}$ propose an actor oriented control system design approach, where the design acknowledges the variety of interaction models among components, and express these interaction styles independently from the functionality of components. In, ${ }^{7}$ Müller-Glaser et al categorize the existing strategies for integration into a number of approaches, including co-simulation, code integration, model encapsulation, and model translation. The author of this paper extends to propose a two-way integration strategy. ${ }^{8}$ Generally speaking, these strategies have their own challenges, such as the synchronization of the simulation processes, auto code generation, and so on.

In this paper, we present an interactive design and simulation platform for flight vehicle systems development. The proposed platform adopts the co-simulation integration concept and avoids code generation challenges faced by other integration approaches. It enables the component design "plug-and-play" in a systems simulation environment, therefore, to bring the "systems simulation" into the "control design" for integrated development. Moreover, the smooth interactive design and simulation is achieved by an adaptive "connect-and-play" capability. As a case study, this paper demonstrates an interactive development between an aircraft pitch tracking control design and flight simulation on a generic jet flight training device (FTD).

The rest of this paper is organized as follows. In Section 2, we describe the interactive design and simulating platform in details. In Section 3, we present the experimental test bed for the integrated flight vehicle systems development, followed by implementation (Section 4) and an interactive flight control design and simulation example (Section 5). Finally, Section 6 offers the concluding remarks. 


\section{Interactive Design and Simulation Platform}

Modern aircraft include a variety of automatic control systems that aid the flight in navigation, flight management, and augmenting the stability characteristics of the airplane. ${ }^{9}$ The aim of flight control systems (FCS) development is to find a solution, given the inputs and desired outputs or tolerable errors, and to integrate design into a functional system that performs its assigned tasks satisfactorily. The FCS development process is typically broken down into several chronological stages that are extensively interrelated and interconnected, ${ }^{10}$ including the following critical ones: 1) Establishment of System Purpose and Overall System Requirements; 2) Detailed Component Design and Selection; and 3) Integration, Testing, and Validation. Due to its complexity, a much more integrated design process may be a natural choice of solution, where interactions among subsystems and components, interactions at different levels of systems complexity, and interactions across different phases can be accounted for, to reduce design iterations and become more robust and reliable. On the one hand, the design of different control channels are independent activities, with customized flight equations that capture the characteristic dynamic behavior associated with that specific channel, and with simplified interacting component models to minimize the coupling effects in control. On the other hand, the final designed controllers need to be implemented and evaluated under a realistic simulation environment, consisting of highfidelity nonlinear aircraft systems models. The design is often tested by a series of systems simulations, including those performed on a flight simulator. In this paper, we propose an interactive design and simulation platform for flight vehicle systems development, especially for flight control systems design and verification.

The integrated development process is illustrated in Figure 1. "Controller Design" refers to iterations of design and design modifications, including multi-paradigm models. The "systems simulation" part implies various levels of simulations as verification and validation methods, including heterogeneous platforms.

There are several integration strategies. ${ }^{7}$ The popular approach in flight control systems development follows the concept of code encapsulation in principle. As shown in Figure 2(a), the controller is designed and validated in isolation by desktop off-line simulation. The controller algorithm codes are generated (in $\mathrm{C}$ code as one example). Then, encapsulated as a monolithic submodel, it is integrated into the model of enclosing system for systems validation and verification. Such process presents the part from "Controller Design" to "Systems Simulation" of the integrated process in Figure 1. In our paper, ${ }^{8}$ we extended the approach by further suggesting a two-way integration strategy, as shown in Figure 2(b). This approach differs from the one-way code encapsulation approach (Figure 2(a)) in that it includes the hardware-in-the-loop, heterogeneous simulations, and it enables "Systems 


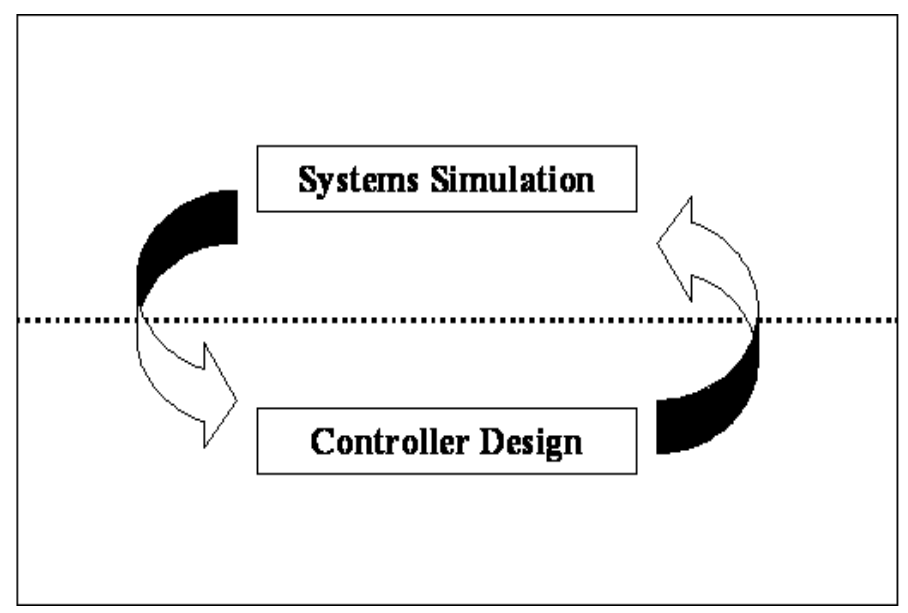

Figure 1. Integrated (FCS) Development Process

Simulation" $\rightarrow$ "Controller Design" capability in the integrated process (Figure 1).

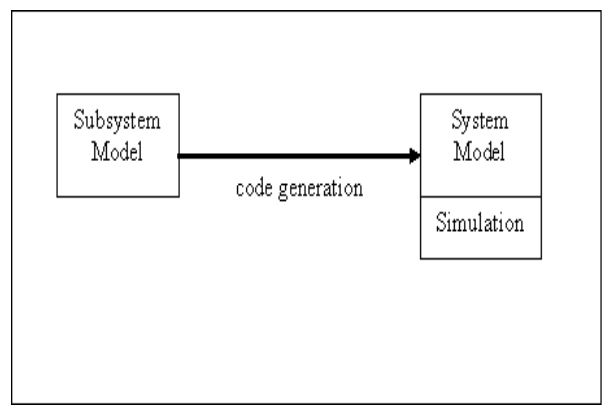

(a) one way code encapsulation

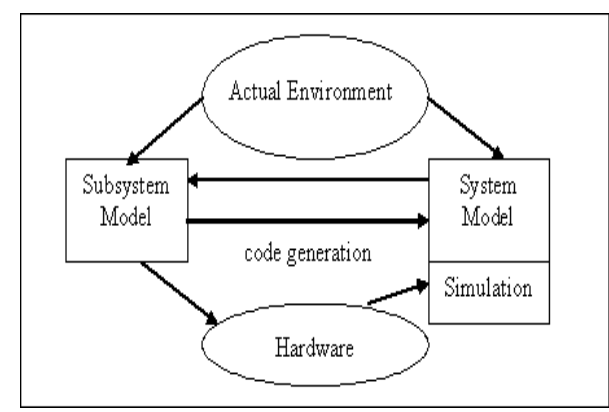

(b) two way code encapsulation

Figure 2. Code-Generation Based Integration Approaches

The challenges of the code-generation based integration approaches include the level of automation, compatibility and synchronization of the models and simulations. Since design codes are used as the media, one needs to make sure that the generated codes can work on heterogeneous simulation platforms, with proper interfaces. If so, it is expected that the code generation can be processed automatically, to avoid tedious manual labour and errors. These challenges are still open research topics. In this paper, instead, we adopt a different integration strategy that is similar to co-simulation in principle. As shown in Figure 3, this proposed interactive platform allows the component model to be simulated (plug-and-play) in a different, system-level environment. Moreover, the platform is adaptable such that the systems simulator can "connect" to the design model directly. We believe that this "connectand-play" capability is one significant improvement over the "plug-and-play" capability. Since there is only one physical design model that takes residence at the component level, 
one can work with this model to make modifications and perform testing "on-line" without the intermediate code-generation process. Obviously, the "connect-and-play" property and adaptability make the design and simulation platform truly interactive and integrated in development.

Under the proposed platform (Figure 4), the integrated flight control system results in a multi-paradigm control framework. It represents a standard flight control system block diagram with some special features.

- The blocks with a drop-down shadow represent "swapping" features. The guidance/command block represents the flight path generation (guidance) or command inputs (for controller design). The actuation and sensor blocks can be replaced by software modules with different levels of fidelity, or even hardware equipments. The vehicle dynamics module can also be replaced by different software modules for different simulation purposes. A simplified linearized dynamics model is used for control system design, while full-scale nonlinear flight equations will be used for high-fidelity simulations, such as flight simulations.

- The blocks inside HIL can be replaced by hardware equipments for hardware-in-theloop (HIL) experimentation.

- The whole flight control system structure, when interacting with other flight systems, can be integrated into a flight simulator for flight simulation, or pilot-in-the-loop (PIL) simulation, to validate the design.

- In order to emulate the reality that different flight systems components are physically installed in different locations and their interactions are communicated through mechanical links or electrical bus, the proposed framework allows for a distributed modeling structure. Each block can be individually modeled, as one software module in different processors. Therefore, it is possible to distribute different parts of a computing task across individual processors operating at the same time, or "in parallel", and thus reduce the overall time to complete the task. Further, the distributed modeling structure makes it feasible to "swap" different modules of the same block, including the hardware-in-the-loop simulation.

- Due to the distributed modeling and "swapping" feature, it is possible to replace block modules developed under different platforms, and even to run simulations on machines from different manufacturers. Therefore, the proposed framework supports heterogeneous simulations.

- The framework not only allows for distributed modeling, but also enables real-time simulation, where interactions and synchronizations among subsystems or components act and react in clock time, as it happens in a real flight environment. 


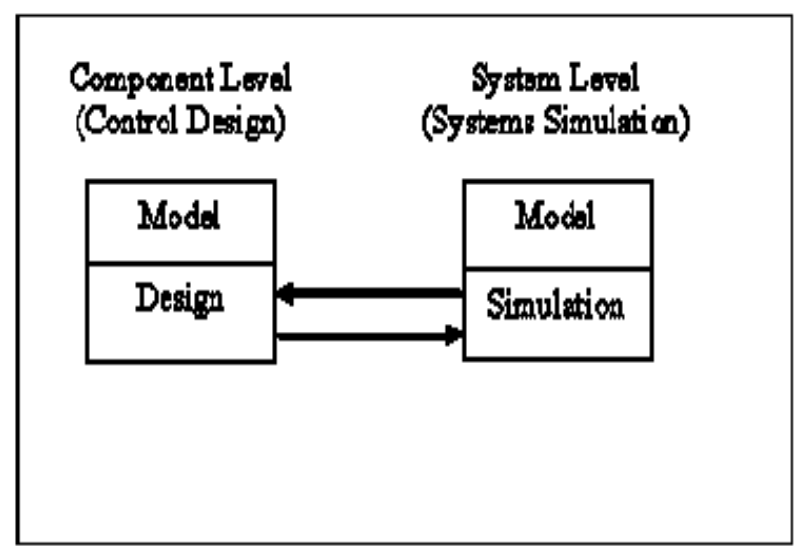

Figure 3. Interactive Design and Simulation Platform

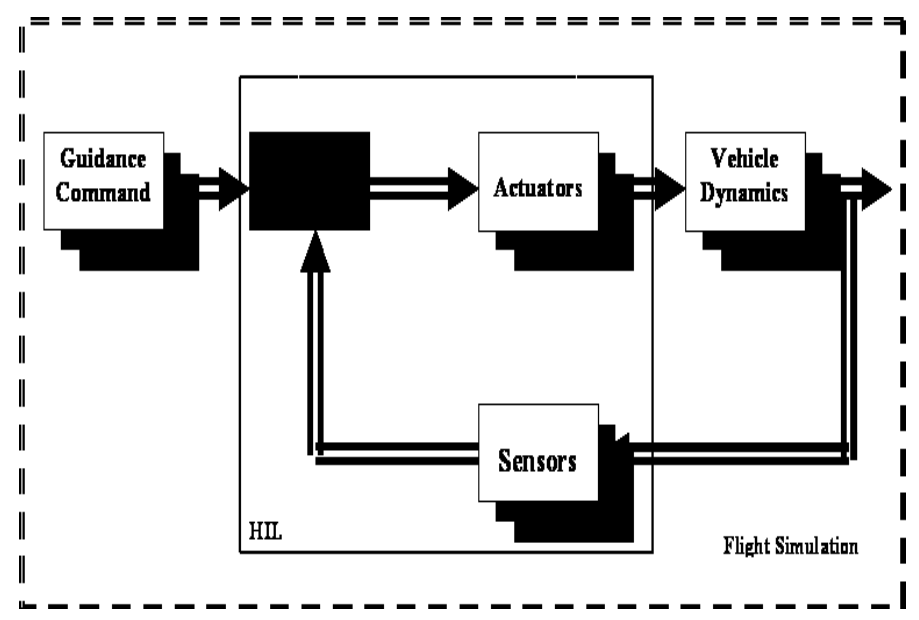

Figure 4. Multiparadigm FCS Block Diagram 
In order to demonstrate the proposed FCS framework and the interactive design and simulation platform, an experimental test bed is set up. A real-time systems simulator and a flight training device (RTSS-FTD) are equipped to provide a suitable proof-of-concept facility, and illustrated by a pitch tracking control example of a generic jet airplane in its cruise condition.

\section{RTSS-FTD Test Bed}

\section{A. The Real-Time Systems Simulator (RTSS)}

The real-time systems simulator (RTSS) facility is a networked cluster of high-end commercial off the shelf (COTS) computers as shown in Figure 5(a). Its core computing features include: three (3) host computers each has dual-Pentium-processors running Windows 2000 OS; four (4) real-time computers each has dual-Pentium-processors running QNX real-time operating system; the real-time nodes are directly connected by 400Mbit/sec FireWire and communicate with hosts over a dedicated $100 \mathrm{Mbit} / \mathrm{s}$ Ethernet network. Further, the system consists of 108 multiple channels IO system for hardware-in-the-loop simulation. The RTSS is also connected through a $1.25 \mathrm{~Gb} / \mathrm{sec}$ Giganet to a similar facility to share data and sources, and it is connected to a 56-alpha-processor high power computer for off-line computing and simulation, as well as data storage. This configuration provides the following key capabilities to support our proposed framework:

- Flexibility. The models are distributed and executed over a network of high-end computers interconnected with a fast real-time communication system; the data is realtime acquired, logged, and stored; the model parameter values are allowed to modify at runtime from a graphical interface; the interconnection with the commercial I/O board is located inside one host computer, allowing for hardware-in-the-loop simulation.

- Development. Matlab/Simulink ${ }^{\mathrm{a}}$ provides a full integration with visual simulation and $\mathrm{C}$ code generation; the model separation is automatic in several interconnected subsystems; the automatic code generation and object code loading take into account of all necessary processors and I/O synchronizations; a library of Simulink icons can easily connect commercial I/O boards to the dynamic models; an application programming interface (API) provides a user-friendly interface to allow for the control of the simulator, the on-line parameter control and results display; it also generates the source codes and typical I/O drivers and real-time modules allowing the user's addition, specialization, and customization.

\footnotetext{
${ }^{\mathrm{a}}$ www.mathworks.com
} 
- Scalability. The system takes advantage of COTS hardware and components to fit the application requirements, and to expand the computing power if necessary.

- Performance. Fire Wire 400-Mbits/second real-time serial bus offers a very low latency for models with loop time as low as $200 \mathrm{~ms}$. The scheduler overhead is less than $10 \mathrm{~ms}$ on a Pentium $233 \mathrm{MHz}$ processor. The minimum loop time on a distributed CPU system is about $80 \mathrm{~ms}$ to account for data synchronization and TCP/IP communication with the host computer. The use of QNX proves a $2 \mathrm{~ms}$ interrupt respond time and a 6 ms context switching time.

\section{B. The Generic Jet Flight Training Device (G-FTD)}

A separate flight training device (FTD) is also set up for flight simulation, as shown in Figure 5(b). This state-of-the-art research simulator simulates the operation of a generic jet aircraft within the tolerances and conditions set out by the Transport Canada Authority. The block diagram of Figure A is a general overview of the system layout. The major aircraft subsystems include the automatic controls, the auxiliary power unit (APU), doors, the engine indication and crew alerting system (EICAS), the electrical systems, the environmental control systems, flight controls and flight instruments, the fuel, pneumatic and hydraulic systems, the landing gear, the lighting, and the navigation and communications systems. The design of the FTD is such that all the simulated functionality is concentrated in the software model running on the host computer. This software model contains all the mathematical and logic modeling to make the FTD behave likes the Generic Jet aircraft. All the other computers and hardware are input/output (I/O) interfaces between the pilot/copilot and the model software running on the host computer. The control loading is handled by a PC on the network. It communicates with the host on the Ethernet switch. This computer has digital wiring running to the primary flight controls in the cockpit. The computer systems are networked through a 100Mbaud Ethernet switch. All the simulated aircraft panels are intelligent; they each contain an embedded CPU which manages their local IO and communicates with the host computer through a CAN bus network. The aircraft flight and subsystem models are developed using $\mathrm{C}$ language. The visual database is developed using the MultiGen paradigm. The control system is developed under the Matlab/Simulink platform.

\section{The RTSS-FTD Connection}

In summary, the RTSS is able to simulate the aircraft systems and flight maneuvers. The features of reconfigurability, modeling and customization of cockpit displays are critical to our systems integration research. Matlab/Simulink is the software development platform. 


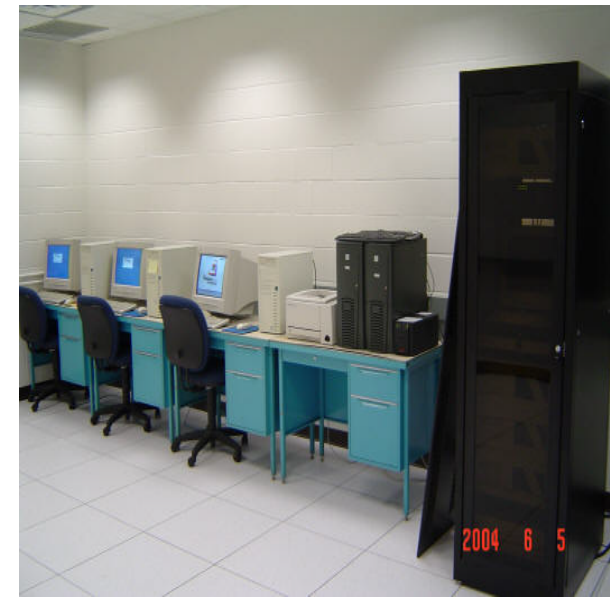

(a)

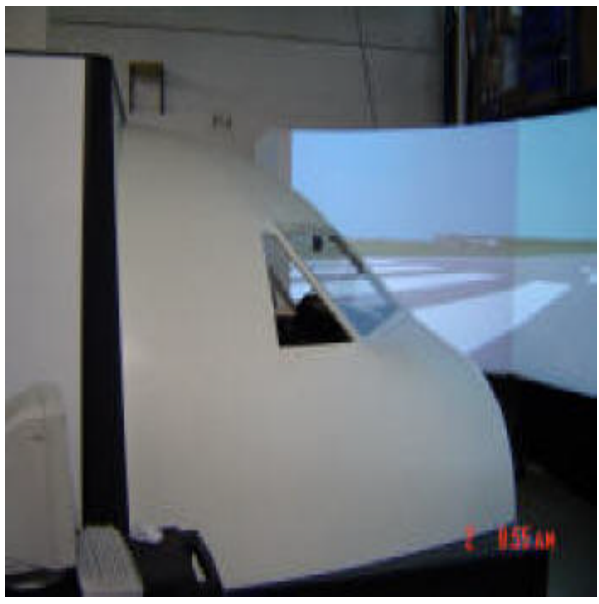

(b)

Figure 5. RTSS-FTD Facilities

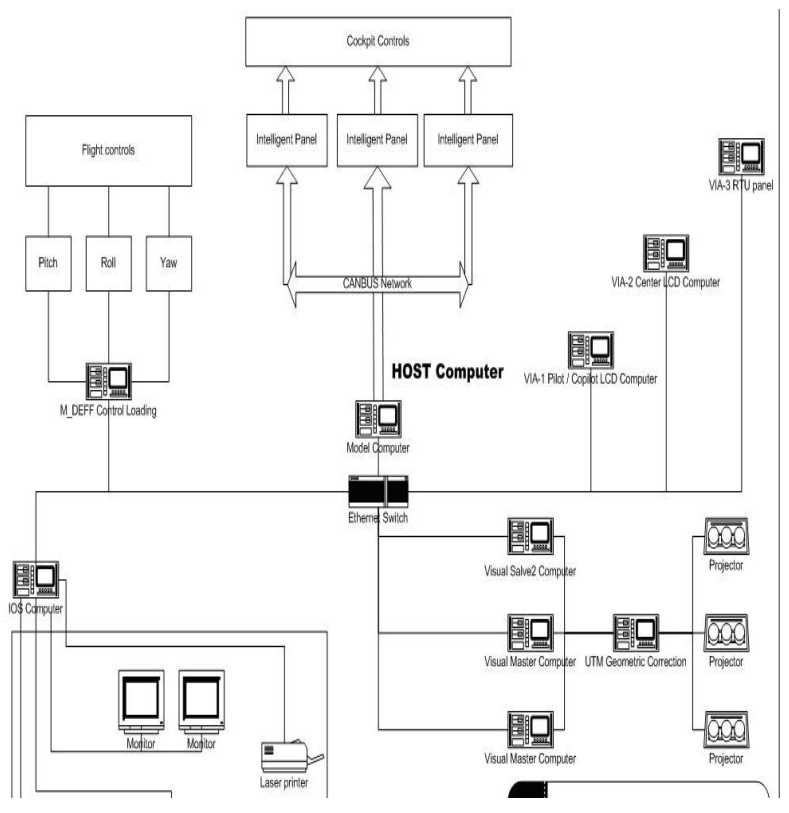

Figure 6. G-FTD Architecture 
The G-FTD presents a more complete and realistic aircraft model, which includes factors not taken into account in the RTSS development. It offers a different perspective as the flight mission may be observed from a cockpit with out-the-window visual and instrument displays. The RTSS and FTD facilities are connected through Ethernet cables to form a networked RTSS-FTD test bed for integrated modeling and simulation activities.

\section{Implementation of Interactive Platform}

The FTD consist of a number of computers with highly specialized tasks. The core of the system is formed by the Model computer, which is responsible for the overall aircraft model, and the Control Loading computer. Three Visual Instruments Aid (VIA) computers are in control of the avionics instrument panels. Three Visual computers are in charge of evaluating the visual databases, each one creating an image of the current view outside the cockpit. Those images undergo blending and geometric correction algorithms in the Universal Transverse Mercator (UTM) correction computer and are finally projected onto a single curved screen. The simulator can be controlled from the Instructor Operation Station (IOS) computer. The Model and Control Loading computers, subjected to hard real-time constraints, run the real time operating system QNX, while all other computers have Windows 2000 installed. This system architecture forms a separated local area network. The IOS computer, however, has two network cards installed, allowing for the possibility to connect the FTD to another network. Figure 7 gives an overview of this layout.

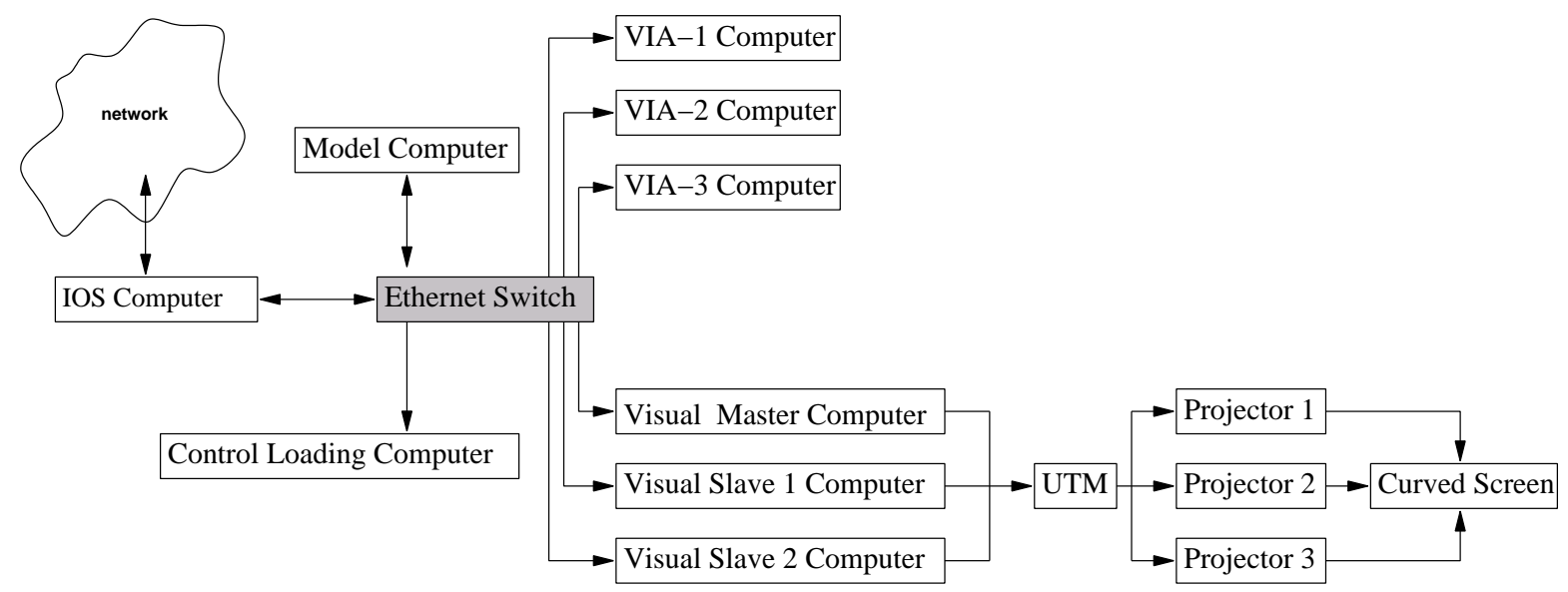

Figure 7. FTD network layout

In order to use the FTD as a test bed for interactive controller design and simulation, a network connection is established for "connect-and-play". The idea is to let an S-function for Matlab/Simulink work as a network I/O-layer, which outputs the current state vector of the FTD and takes control commands as inputs, as it is commonly done with hardware-in-the- 
loop approaches (Figures 8 and 9). Note that the control input can carry additional payload, if necessary. In particular, the S-function allows for three values of wind components, which, if given, will be used to overwrite the built-in wind model.

The communication link between a Matlab/Simulink environment and the FTD hard/software has to fulfill a number of requirements. Mainly, the interface should be

- easy to use no compilation should be required when switching between the built-in and a remotely controlled autopilot

- robust the communication should not break down on minor network problems

- fast the packets should have low overhead to improve network speed

- capable of real time maximum response times should be guaranteed

- extensible it should be easy to integrate additional payload

- portable the packet format should not depend on operating system peculiarities

It is then decided that only a TCP/IP connection offers the robustness needed for controller operation. The packet format used for the network connection is simple, yet extensible. A fixed-length integer specifying the total packet length is followed by an arbitrary number of triples specifying an identifier, a value and a delimiter. The S-function is written in the programming language $\mathrm{C}$, and can be further customized at compile time using preprocessor macros. This way, among other preferences, maximum allowable packet length and floating point data formats can be adjusted.

In order to guarantee maximum response times of the network connection, network timeouts have been implemented, also settable with $\mathrm{C}$ preprocessor macros. If a timeout occurred, predefined hard coded default values are assumed. In this case, the user is provided with a warning.

One of the demands for the connection is that it should be possible to switch between the built-in and a remote controlled autopilot "on the fly". This required the logic depicted in Figure 10. In practice, a custom autopilot takes over as soon as a Simulink simulation containing the network S-function is started, while in general, the built-in autopilot is in place while the simulation is not running.

\section{A Pitch Tracking Example}

In this section, a pitch tracker for a jet airplane at its cruise condition is considered, with elevator deflections being used as the control input. In early design stages for aircraft 
Controller, implemented in Matlab/Simulink, running on any computer inside the FSC network.

Uses information about current state to calculate

appropriate control commands.

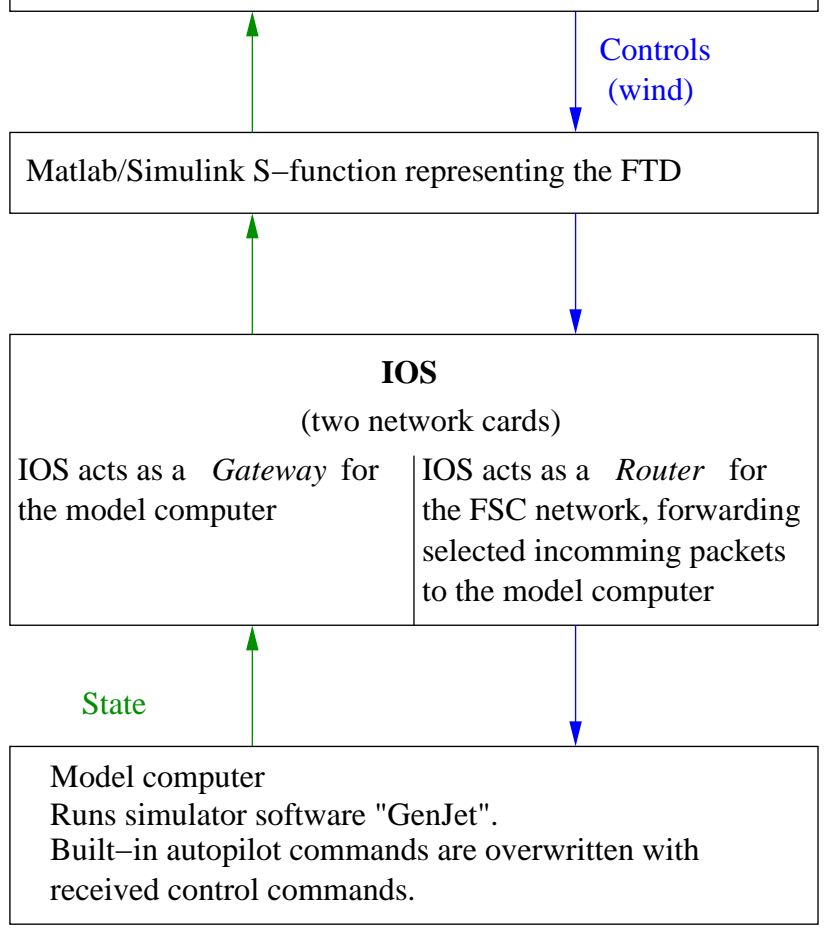

Figure 8. Network communication

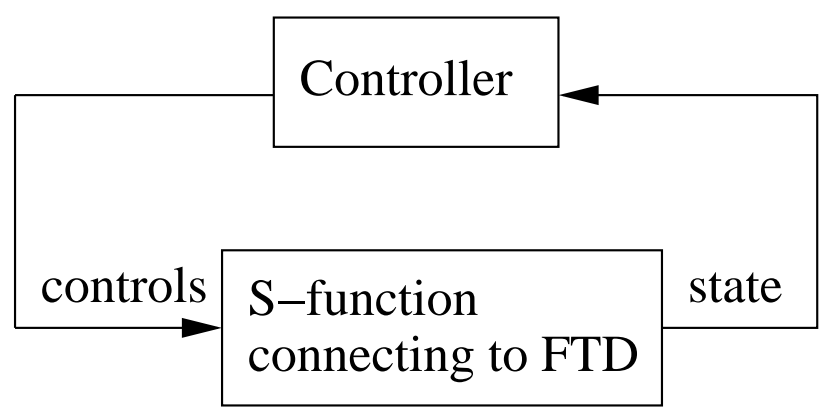

Figure 9. Integration in Matlab/Simulink 


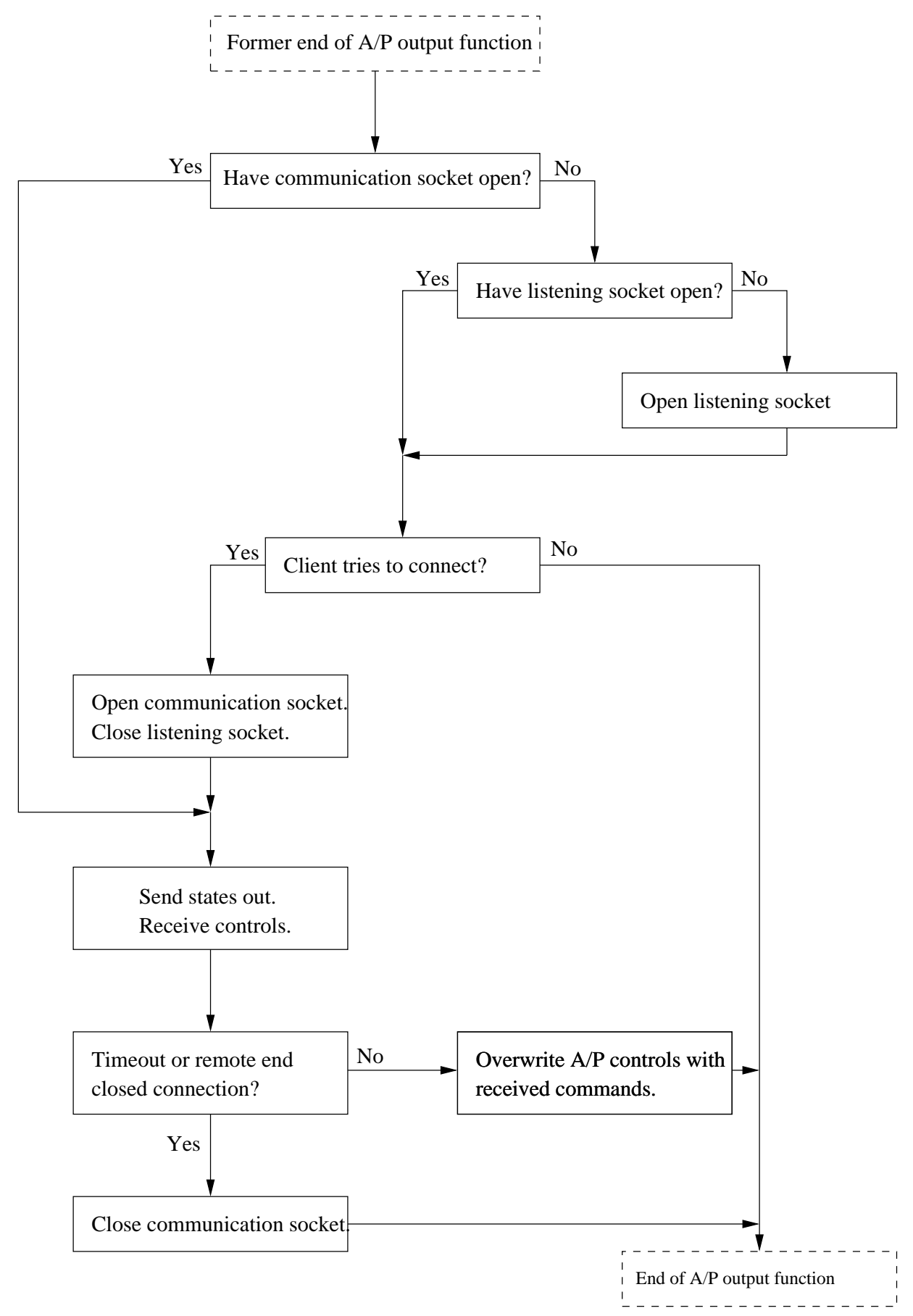

Figure 10. Connection logic 

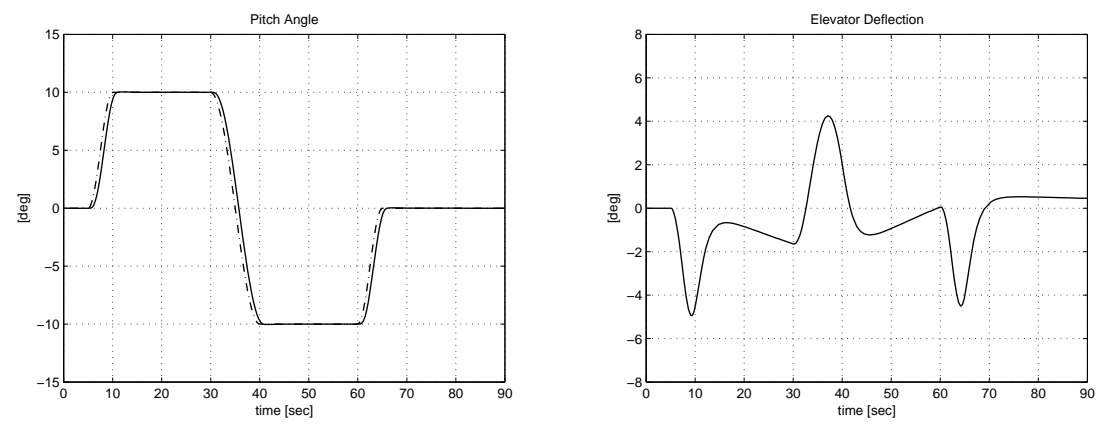

Figure 11. Off-line Simulink simulation, no actuator dynamics
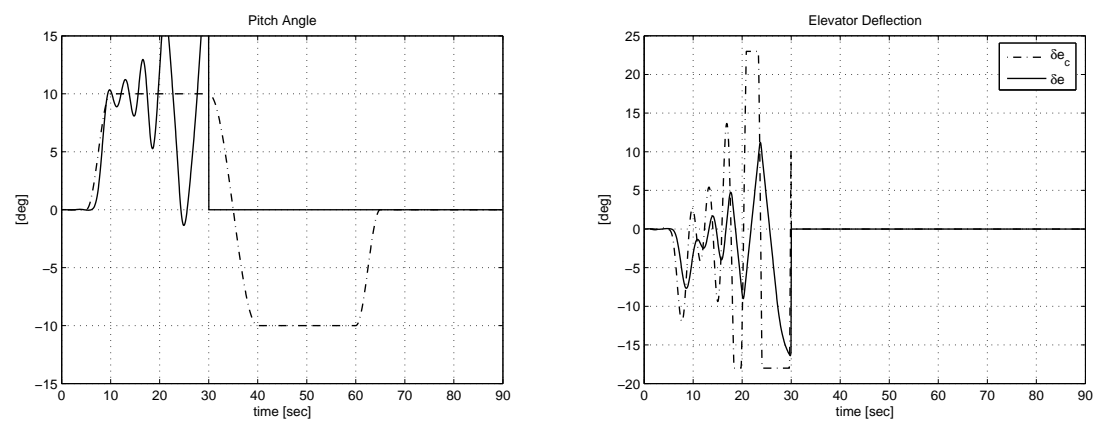

Figure 12. FTD in the loop simulation, no actuator dynamics
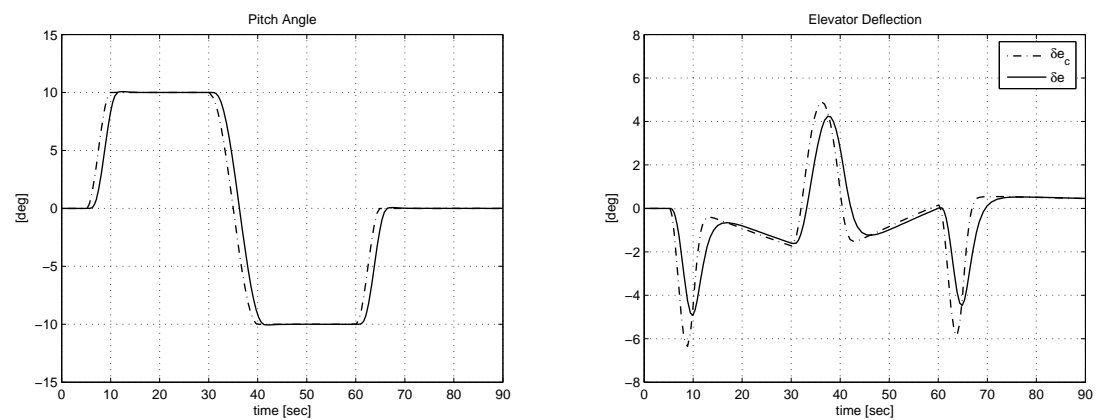

Figure 13. Off-line Simulink simulation, re-designed controller
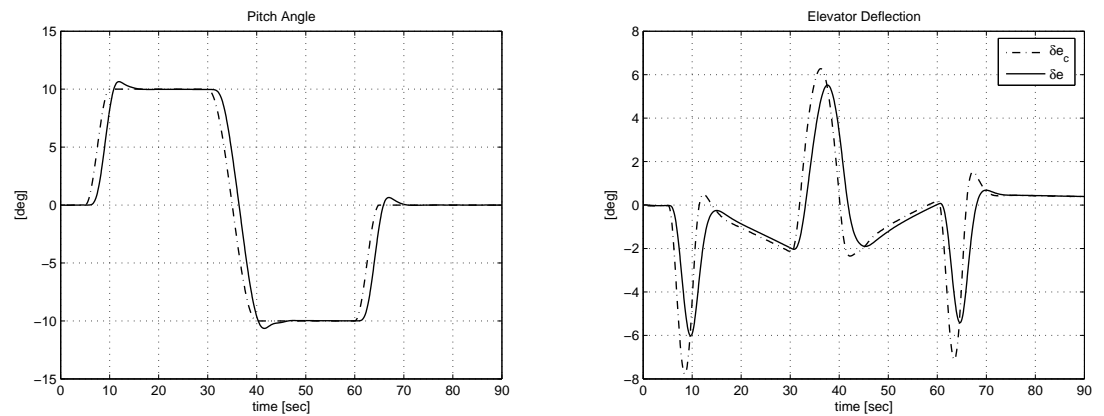

Figure 14. FTD in the loop simulation, re-designed controller 
controllers, the influence of actuator dynamics is oftentimes neglected. In this case, however, this turns out to be an unjustifiable simplification. Figure 11 shows the simulation results of a LQR based pitch tracker, acting on a linear aircraft model with unmodeled actuator dynamics. On the left side, the pitch angle (solid line) follows the trajectory (dashdot line) nicely, while the control effort, depicted on the right, is rather small. When this controller is tested with the FTD in the loop, it becomes unstable; the simulation shown in Figure 12 has to be aborted after 30 seconds. On the right side, the commanded elevator position $\delta e_{c}$ is plotted as a dashdot line against the current elevator position $\delta e$. This leads to the assumption that the instability is caused by the time-delay introduced by actuator motion. Therefore, the actuator was modeled as a first-order lag filter, and included in the linear aircraft model during controller re-design. Simulation results with the linear model and the FTD in the loop are shown in Figures 13 and 14. Although Figure 14 still shows a slight overshoot, the improvement over Figure 12 is obvious.

In this example, we demonstrate that the flight control design and its verification achieve the expectation by using our proposed interactive (control) design and (flight) simulation platform. Design modifications or even redesigns are quick to perform since the simulation directly "call" the component that still takes residence at the local control system development environment (Matlab/Simulink). The design impact on overall system performance is also easy to obtain by the interactive platform due to its "connect-and-play" capability. One doesn't need to go through the code generation and interfacing process.

\section{Conclusions}

In this paper, an interactive design and simulation platform was presented. Its "connectand-play" capability and adaptability enabled "on-line" interaction between design and simulation during the integrated development. The implementation of the proposed platform was addressed in details, and an aircraft flight control system development example was given as a demonstration on an experimental RTSS-FTD test bed. Multi-paradigm models and heterogeneous simulations were integrated through a TCP/IP network of I/O. This platform is deemed to attract interests in development due to its simple strategy. The interaction and integration can be achieved without "re-inventing" machine or architecture.

\section{References}

\footnotetext{
${ }^{1}$ Heck, B. S., Wills, L. M., and Vachtsevanos, G. J., "Software enabled control: background and motivation," Proceedings of the American Control Conference, Arlington, VA, June 25-27 2001, pp. 3433 3438 .

${ }^{2}$ Wills, L., Sander, S., Kannan, S., Kahn, A., Prasad, J., and Schrage, D., "An open control platform
} 
for reconfigurable, distributed, hierarchical control systems," Proceedings of the Digital Avionics Systems Conference, Philladephia, PA, October 2000.

${ }^{3}$ Paunicka, J. L., Mendel, B. R., and Corman, D. E., "The OCP - an open middleware solution for embedded systems," Proceedings of the American Control Conference, Arlington, VA, June 25-27 2001, pp. $3445-3450$.

${ }^{4}$ Mosterman, P. J. and Vangheluwe, H., "Guest Editorial: Special Issue on computer automated multiparadigm modeling," ACM Transactions on Modelig and Computer Simulation, Vol. 12, No. 4, October 2002, pp. 249-255.

${ }^{5}$ Mosterman, P. J., Sztipanotits, J., and Engell, S., "Computer Automated Multiparadigm Moldeing in control systems technology," IEEE Transactions on Contorl Systems Technology, Vol. 12, No. 2, March 2004, pp. 223-234.

${ }^{6}$ Liu, J., Eker, J., Janneck, J. W., Liu, X., and Lee, E., "Actor-oriented control system design: a responsible framework perspective," IEEE Transactions on Contorl Systems Technology, Vol. 12, No. 2, March 2004, pp. 250-262.

${ }^{7}$ Müller-Glasser, K. D. and Frick, G., "Multiparadigm Modeling in embedded systems design," IEEE Transactions on Contorl Systems Technology, Vol. 12, No. 2, March 2004, pp. 279-292.

${ }^{8}$ Liu, H. H., "Multiparadigm design, validation and verification by simulation in flight control system development," Proceedings of IEEE Conference on Computer Aided Control Systems Design, Taipei, Taiwan, ROC, September 2-4 2004, pp. 71-76.

${ }^{9}$ Nelson, R. C., Flight Stability and Automatic Control, WCB McGraw-Hill, 2nd ed., 1998.

${ }^{10}$ McRuer, D., Ashkenas, I., and Graham, D., Aircraft Dynamics and Automatic Control, Princeton University Press, 1973. 